\title{
Controle social na gestão do SUS: aspectos estruturais, organizativos e estratégicos
}

\author{
Social control in SUS management: structural, organizational and strategic aspects \\ EI control social en la gestión del SUS: aspectos estructurales, organizativos y estratégicos
}

Recebido: 10/02/2022 | Revisado: 18/02/2022 | Aceito: 23/02/2022 | Publicado: 05/03/2022

\author{
José Felipe de Freitas Gomes \\ ORCID: https://orcid.org/0000-0001-9096-6930 \\ Fundação Universidade Federal de Rondônia, Brasil \\ E-mail: gomesjf@hotmail.com \\ Nathalia Halax Orfão \\ ORCID: https://orcid.org/0000-0002-8734-3393 \\ Fundação Universidade Federal de Rondônia, Brasil \\ E-mail: nathaliahalax@unir.br
}

\begin{abstract}
Resumo
A inserção da população na definição das políticas públicas de saúde é considerado um dispositivo de consolidação da democracia participativa, na qual representa a efetivação de um dos princípios do SUS. Objetivou-se analisar o funcionamento, estrutura e organização do controle social na gestão do SUS. Trata-se de uma revisão integrativa realizada a partir da estratégia PICo, a qual contribuiu na elaboração da pergunta norteadora e seleção dos descritores no DeCS, Emtree e MeSH. A busca nas bases de dados da Lilacs, PubMed, Embase, Scopus e Web of Science ocorreu, em novembro de 2020, considerando os artigos completos, publicados a partir da criação do SUS até 2020, nos idiomas português, espanhol e inglês, e que não estavam duplicados. Foram selecionados 66 artigos que versavam sobre os aspectos organizativos, estruturais e de funcionamento dos órgãos colegiados de controle social e gestão participativa, os quais refletem no desenvolvimento de suas práticas, sobressaindo as vivências dos Conselhos de Saúde no que se refere às estratégias de capacitação de seus integrantes. Tais experiências maximizam a consolidação de uma consciência cidadã e crítica, favorecendo ainda aos distintos sujeitos coletivos uma participação efetiva e de qualidade, materializando-se como uma legítima ferramenta de gestão pública.
\end{abstract}

Palavras-chave: Gestão do SUS; Controle social; Participação popular.

\begin{abstract}
The inclusion of the population in the definition of public health policies is considered a device for the consolidation of participatory democracy, in which it represents the realization of one of the principles of the SUS. The objective was to analyze the functioning, structure and organization of social control in the management of the SUS. This is an integrative review based on the PICo strategy, which contributed to the elaboration of the guiding question and selection of descriptors in DeCS, Emtree and MeSH. The search in the Lilacs, PubMed, Embase, Scopus and Web of Science databases took place in November 2020, considering the complete articles, published from the creation of the SUS until 2020, in Portuguese, Spanish and English, and which were not duplicated. Sixty-six articles were selected that dealt with the organizational, structural and operational aspects of collegiate bodies for social control and participatory management, which reflect on the development of their practices, highlighting the experiences of Health Councils with regard to training strategies for its members. Such experiences maximize the consolidation of a citizen and critical conscience, also favoring the different collective subjects an effective and quality participation,
\end{abstract} materializing itself as a legitimate tool of public management.

Keywords: SUS management; Social control; Popular participation.

\section{Resumen}

La inclusión de la población en la definición de las políticas públicas de salud es considerada un dispositivo para la consolidación de la democracia participativa, en la que representa la realización de uno de los principios del SUS. El objetivo fue analizar el funcionamiento, estructura y organización del control social en la gestión del SUS. Esta es una revisión integradora basada en la estrategia PICo, que contribuyó a la elaboración de la pregunta guía y selección de descriptores en DeCS, Emtree y MeSH. La búsqueda en las bases de datos Lilacs, PubMed, Embase, Scopus y Web of Science se realizó en noviembre de 2020, considerando los artículos completos, publicados desde la creación del SUS hasta 2020, en portugués, español e inglés, y que no estuvieran duplicados. Fueron seleccionados 66 artículos que trataban los aspectos organizativos, estructurales y operativos de los órganos colegiados de control social y gestión participativa, que reflexionan sobre el desarrollo de sus prácticas, destacando las experiencias de los Consejos de Salud en lo que respecta a las estrategias de formación de sus miembros. Tales experiencias potencian la consolidación de una conciencia ciudadana y crítica, favoreciendo además a los diferentes sujetos colectivos una participación efectiva y de calidad, materializándose como una legítima herramienta de gestión pública.

Palabras clave: Gestión del SUS; Control social; Participación popular. 


\section{Introdução}

A participação popular, estabelecida constitucionalmente, foi uma conquista da população resultante de significativas lutas dos movimentos sociais, com destaque ao Movimento da Reforma Sanitária - que sugiram após o período ditatorial (Coelho, 2012; Oliveira et al., 2015) - caracterizado pela luta a favor da democratização na saúde (Aciole, 2007) e redemocratização do país (Durán et al., 2014; Gerschman, 2004; Pessoto, Nascimento et al., 2001), contrário, portanto, ao autoritarismo e aos regimes centralizadores (Mittelbach et al., 2014; Vieira et al., 2011).

No Brasil, a expressão "controle social" dependendo do momento histórico, pode ser conceituado de maneiras distintas. No período ditatorial, por exemplo, esse termo era caracterizado como o controle do Estado sobre a sociedade, inviabilizando qualquer tipo de expressão da população (Cotta et al., 2009; Silva, Cruz et al., 2007; Vilaça et al., 2019). Por outro lado, após o Movimento da Reforma Sanitária, o termo “controle social” passou a ser entendido como a prática de cogestão pública da sociedade, por meio de seus representantes, sobre as ações estatais (Batagello et al., 2011; Cotta et al., 2009; Duarte et al., 2012; Oliveira, 2004; Oliveira et al., 2009; Oliveira, Ianni et al., 2013; Shimizu et al., 2013; Silva et al., 2007).

O controle social, fato contemporâneo na conjuntura histórica do país (Vilaça et al., 2019), é, então, considerado uma conquista da sociedade brasileira que favorece o estabelecimento de práticas democráticas e descentralizadoras (Cotta et al., 2009), possibilitando a materialização da gestão compartilhada e participativa (Oliveira et al., 2009; Severo et al., 2012). Nesse sentido, a participação da comunidade é um princípio construído através de uma ativa cultura política democrática, que, rompendo com práticas hegemônicas de poder e autoritarismo, desencadeia um movimento de corresponsabilidade entre os distintos sujeitos sociais envolvidos (Cotta et al., 2010).

Porém, para alcançar essa realidade, é imprescindível que exista o pleno funcionamento das instâncias colegiadas do Sistema Único de Saúde (SUS) - Conferências e Conselhos de Saúde - que, embora se apresentem em processo de formação, podem ser considerados instrumentos sociais fundamentais no desenvolvimento da descentralização e democratização das políticas públicas na saúde (Oliveira et al., 2013).

As Conferências de Saúde são espaços deliberativos institucionalizados e representativos de participação social (Müller et al., 2014), que objetivam avaliar a situação de saúde da comunidade e formular, propor e deliberar diretrizes direcionadas ao estabelecimento de políticas públicas neste setor (Coelho, 2012; Miranda et al., 2016; Shimizu et al., 2009).

Por sua vez, o Conselho de Saúde, com sua pluralização da representação, é considerado pilar institucional do SUS, possuindo natureza paritária, permanente e caráter deliberativo, sendo considerado um espaço público de consolidação da democracia nas diferentes esferas governamentais (Aciole, 2007; Durán et al., 2014; Oliveira et al., 2013; Serapioni et al., 2006).

Atualmente, são mais de 5.600 Conselhos de Saúde no país (Da Silva et al., 2020; Santos et al., 2020), que possuem as funções de formular estratégias, controlar e fiscalizar a execução da política pública de saúde, inclusive nos aspectos econômico-financeiros (Coelho, 2012; Gonçalves et al., 2013; Santos et al., 2020). Dessa forma, essas instâncias participativas, ao mesmo tempo em que promovem o avanço da participação popular em saúde, também proporcionam a democracia social (Aciole, 2003).

A temática da participação da comunidade, nas diversas instâncias participatórias, tem sido objeto de interesse de inúmeros estudiosos (Pessoto et al., 2001), com pesquisas direcionadas principalmente aos Conselhos de Saúde, as quais têm investigado as particularidades da dinâmica dessas instâncias de controle social, assim como os entraves que as mesmas têm enfrentado (Miranda et al., 2016; Rocha et al., 2020). Estudos reforçam a fragilidade técnica e política dos conselheiros de saúde - especialmente o segmento representativo dos usuários - para uma atuação eficiente frente às ações de proposição que constituem práticas rotineiras nos espaços institucionalizados de deliberação (Martinez et al., 2016; Van Stralen et al., 2006). 
Logo, depreende-se que as instâncias deliberativas e paritárias, por apresentarem-se em constante processo de formação, não se apresentam completamente prontas, impedindo a existência de membros (dos distintos segmentos sociais) permanentemente e plenamente capacitados. Diante disso, torna-se necessário compreender como estão estruturadas e organizadas as instâncias de participação social, inclusive de que forma e com qual frequência ocorre a capacitação dos diferentes sujeitos coletivos que integram as arenas de participação, pois considera-se que tais fatores influenciam diretamente na consolidação da cultura participativa e, consequentemente, no fortalecimento (ou não) do controle social no SUS.

Destarte, o presente estudo objetivou analisar o funcionamento, estrutura e organização do controle social na gestão do SUS, bem como descrever as estratégias e periodicidade de capacitação dos conselheiros de saúde nos órgãos colegiados do SUS.

\section{Metodologia}

Revisão integrativa desenvolvida em seis etapas metodológicas: definição do tema e da pergunta norteadora; estabelecimento dos critérios para inclusão e exclusão dos estudos; identificação dos estudos pré-selecionados e selecionados; categorização dos estudos selecionados; análise e interpretação dos resultados; e, por último, apresentação da revisão e síntese do conhecimento (Botelho et al., 2011).

A pergunta norteadora - "Como a literatura tem abordado sobre a participação popular e o controle social na gestão do SUS?" - foi elaborada a partir da estratégia PICo (The Joanna Briggs Institute, 2014), na qual o primeiro elemento da estratégia "P" (problema) consistiu na gestão do SUS; o segundo "I" (fenômeno de interesse) o controle social; e o último elemento "Co" (contexto) considerou a participação popular.

A expressão de busca foi constituída utilizando os operadores booleanos para combinar os descritores indexados e seus respectivos sinônimos dos Descritores em Ciência da Saúde (DeCS), Embase Subject Headings (Emtree) e Medical Subject Headings (MeSH), nos idiomas português, espanhol e inglês, a saber: "Gestão da saúde da população" OR "Política de Saúde" OR "Sistema Único de Saúde" OR "Sistemas de Saúde" OR "Gestão em saúde" OR "Cursos de Capacitação" OR "Educação Continuada") AND (“Conferências de Saúde" OR "Conselhos de Saúde" OR "Políticas de Controle Social”) AND ("Participação da Comunidade" OR "Participação Social" OR "Planejamento Participativo" OR "Controle Social Formal").

A pesquisa ocorreu em novembro de 2020 na Literatura Latino-Americana e do Caribe em Ciências da Saúde (Lilacs), US National Library of Medicine (PubMed), Embase, Scopus e Web of Science via Portal de Periódicos da Coordenação de Aperfeiçoamento de Pessoal de Nível Superior (CAPES), considerando, ainda nos campos de busca - descritores de assunto, palavras do título e resumo.

Os critérios de inclusão foram artigos científicos completos, publicados entre 1988 e 2020, disponibilizados nos idiomas português, espanhol ou inglês, sendo estes adotados nas bases de dados. Posteriormente, os artigos foram exportados para o programa de revisão gratuito da web de versão única chamado Rayyan Qatar Computing Research Institute (Rayyan QCRI), visando identificar os artigos duplicados, o qual foi considerado como um critério de exclusão; bem como leitura e análise dos títulos e resumos dos estudos por dois pesquisadores independentes, cujas discordâncias foram realizadas por um terceiro avaliador.

O critério de elegibilidade adotado foi selecionar os artigos que versavam sobre o funcionamento, estrutura e organização do controle social na gestão do SUS, além das estratégias e periodicidade de capacitação dos conselheiros de saúde nos órgãos colegiados do SUS. Os dados foram extraídos dos artigos selecionados para leitura na íntegra seguindo a recomendação Preferred Reporting Items for Systematic Reviews and Meta-Analyses (PRISMA) (Moher et al., 2009). 


\section{Resultados e Discussão}

Dos 253 artigos científicos encontrados, 187 foram excluídos por não se caracterizarem como artigos completos (47), estarem duplicados (40), não atenderem ao critério de elegibilidade (88) e abordarem na leitura na íntegra outros assuntos (12), tais como as bases socioculturais do controle social envolvendo a saúde indígena, o processo de descentralização do SUS, a vigilância sanitária na agenda do espaço de controle social, os desafios das instâncias colegiadas do SUS, o envolvimento do público e do paciente no processo de incorporação de tecnologias. Totalizando 66 estudos para este estudo (Figura 1).

Figura 1. Fluxograma para seleção dos artigos incluídos nesta revisão de literatura, 2020.

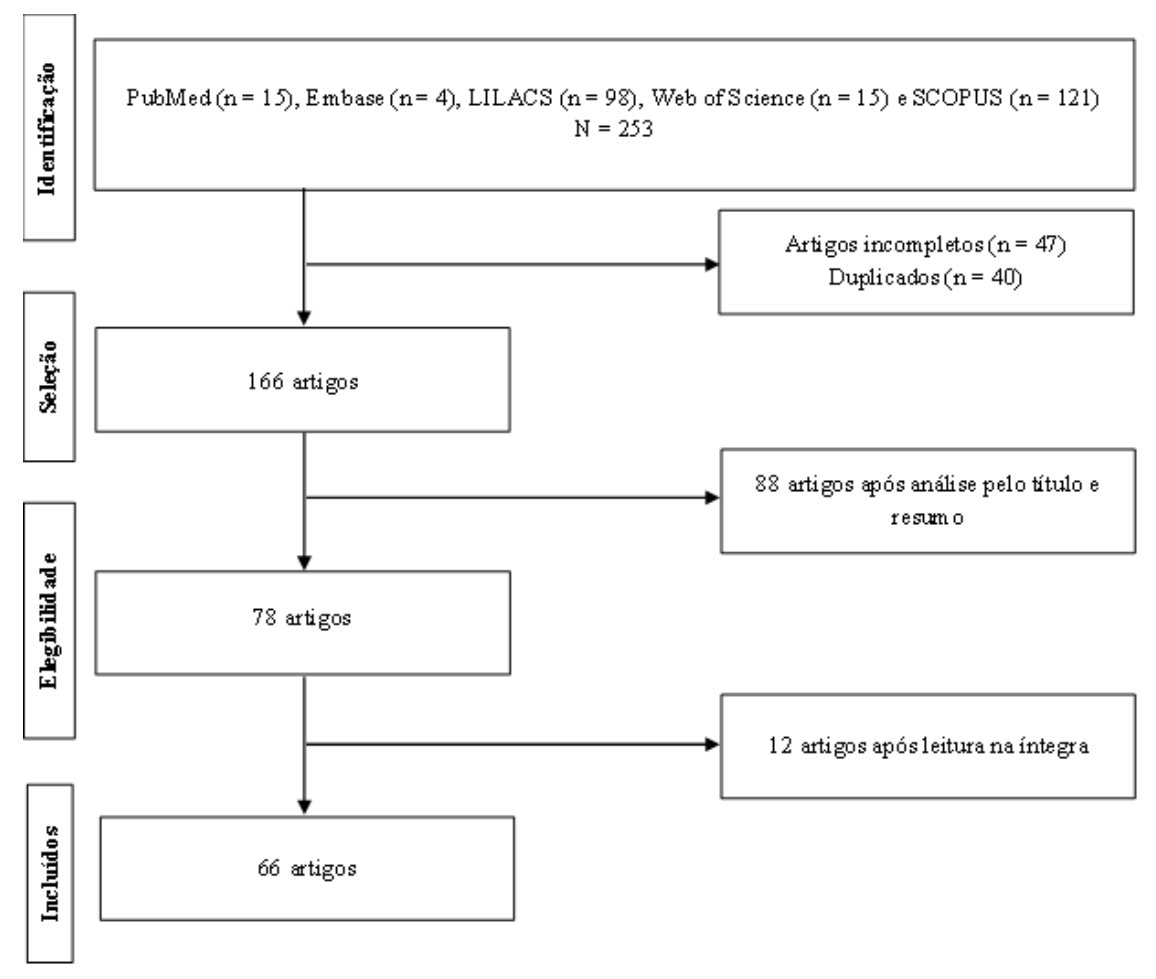

Fonte: Adaptado de Moher et al. (2009).

\section{Estrutura, funcionamento e organização das instâncias colegiadas formais}

O desenvolvimento e a consolidação das instâncias deliberativas são resultantes de sua organização e estrutura interna (Aciole, 2007). Para isso, é fundamental que o regimento interno e demais normas de funcionamento dos órgãos de controle social estejam disponíveis aos conselheiros, com o objetivo de orientar a organização e o funcionamento dos Conselhos de Saúde, conforme identificado por diferentes estudos (Magalhães et al., 2018; Oliveira et al.,2013; Shimizu et al., 2009; Vieira et al., 2011; Zambon et al., 2011), não obstante diversos conselheiros desconheçam os regimentos internos (Cotta et al., 2009) e, consequentemente, os princípios e diretrizes que orientam a estruturação e funcionamento das instâncias participativas das quais fazem parte (Cotta et al., 2011).

Apesar de previsto em Lei o funcionamento dos Conselhos de Saúde através de reuniões mensais e, de forma extraordinária, quando necessário, a maioria está cumprindo com a sua regularidade (Labra, 2008; Magalhães et al., 2018; Rocha et al., 2020; Shimizu et al., 2013; Silva et al., 2019; Van Stralen et al., 2006; Vilaça et al., 2019; Zambon et al., 2011), porém confirma-se que tal diretriz não é uma realidade em outros locais (Bispo Júnior et al., 2006; Cotta et al., 2011; Vieira et al., 2011) que, eventualmente, realizam reuniões com periodicidade semestral (Zambon et al., 2011), a qual pode estar relacionada com inúmeros desafios (Bispo Júnior et al., 2008) que refletem no funcionamento e no fortalecimento do exercício do controle social para a gestão do SUS. 
Determinados espaços colegiados restringem a participação direta dos cidadãos (Van Stralen et al., 2006), enquanto em outras as reuniões são abertas ao público, sendo os encontros divulgados no sítio eletrônico da própria Secretaria de Saúde ou a convocação ocorre mediante calendário previamente estabelecido (Escorel et al., 2008; Moreira et al., 2008; Shimizu et al., 2009; Vilaça et al., 2019), inclusive com as pautas (Shimizu et al., 2009) definidas pelo presidente dos Conselhos ou pela plenária (Van Stralen et al., 2006), bem como por representantes dos segmentos de usuários e trabalhadores da saúde (Silva et al., 2019). Em outro cenário, foi realizado o encaminhamento prévio de pauta e material de apoio aos participantes, incluindo os balancetes contábeis e documentos relacionados à prestação de contas, possibilitando, assim, maior efetividade no monitoramento das finanças por parte dos conselheiros, além de proporcionar a qualificação do debate no colegiado (Rocha et al., 2020).

Ainda sobre o funcionamento das instâncias de participação social, alguns Conselhos de Saúde prezam pela abordagem intersetorial para a variedade de conhecimentos, determinação e competência (Shimizu et al., 2009), embasados no conceito ampliado de saúde, em que se reforça que a interferência nos determinantes sociais de saúde acontece mediante a (re)construção com diferentes segmentos e setores, tais como a educação e cultura (Moura et al., 2017; Oliveira et al., 2013; Zambon et al., 2011).

Em relação à diversidade de segmentos e, por conseguinte, de instituições e categorias representadas nos Conselhos de Saúde, principalmente entre os usuários e trabalhadores (Silva et al., 2019), percebe-se o chamado "pluralismo", responsável pelo fortalecimento dessas instâncias deliberativas. Entretanto, quando algum segmento se sobressai, o pluralismo perde o equilíbrio, ocasionando, por consequência, o enfraquecimento do colegiado (Batista et al., 2010). Logo, as instituições mais organizadas possuem mais voz, tendo em vista que conseguem expor mais efetivamente suas necessidades. Por outro lado, tal organização pode favorecer o direcionamento das prioridades em saúde, assim como dos debates políticos, aos interesses dessas instituições (Moreira et al., 2008).

Quanto à paridade sobre a representação dos distintos segmentos sociais, estabelecida na legislação federal vigente, estipula-se que 50\% dos órgãos colegiados deverão ser constituídos por entidades e movimentos que representem os usuários, 25\% pelos trabalhadores da área da saúde e 25\% por prestadores de serviços e/ ou representantes do governo (Brasil, 2012), conforme identificado na literatura (Batista et al., 2010; Kezh et al., 2016; Moreira et al., 2008; Rocha et al., 2020; Shimizu et al., 2009; Silva et al., 2019; Vilaça et al., 2019).

Por sua vez, tal perspectiva se mostra complexa em outros cenários nos quais detectam-se irregularidades na distribuição de vagas estabelecidas para cada segmento representado (Assis, 2003; Bispo Júnior et al., 2006; Bispo Júnior et al., 2008; Cotta et al., 2010; Cotta et al., 2011; Escorel et al., 2008), de uma forma bastante diversificada (Escorel et al., 2008), em que ocorre, por exemplo, a junção dos segmentos dos trabalhadores da saúde e prestadores de serviços/gestores, formando uma grande categoria denominada de "promotores da saúde", prevista em Lei municipal (Kezh et al., 2016), que, eventualmente, ocasiona práticas indevidas de negociações diretas entre os conselheiros de determinados segmentos sociais e os secretários de saúde (Van Stralen et al., 2006).

Por mais que a paridade entre usuários e demais segmentos seja considerada um indicador do nível de democratização das instâncias de participação social (Zambon et al., 2011), possibilitando que o processo de gestão da saúde alcance as necessidades e desejos da comunidade (Batagello et al., 2011), a distribuição adequada de conselheiros por segmento social, conforme previsto legalmente, não significa a legitimação dos Conselhos de Saúde quanto ao pleno controle social (Duarte et al., 2012; Fakhouri et al., 2016; Landerdhal et al., 2010), da mesma forma que o cumprimento da formalidade de distribuição paritária de membros nas Conferências de Saúde não é garantia de representatividade efetiva (Müller Neto et al., 2014).

Ainda que seja previsto legalmente que, nos Conselhos de Saúde, não é permitida a participação de membros eleitos do Poder Legislativo, bem como representações do Poder Judiciário e do Ministério Público (MP), como conselheiros de saúde 
(Brasil, 2012), verificou-se que essa diretriz nem sempre é respeitada, seja pela presença de vereadores exercendo a atribuição de conselheiros (Cotta et al., 2011) e/ ou devido ao envolvimento direto de representantes políticos nas instâncias participativas, podendo resultar em inibição do posicionamento crítico dos conselheiros (Bispo Júnior et al., 2008).

Acerca do fortalecimento do exercício do controle social na política de saúde, os órgãos colegiados podem estabelecer vínculos com instituições de apoio à participação popular, através da ampliação dos diálogos e do compartilhamento das ações (Zambon et al., 2013), ainda que, conforme citado, os integrantes das mesmas não possam exercer o papel como membros dos Conselhos. Assim sendo, os Conselhos de Saúde devem estabelecer integração com instituições do controle externo, tais como o Tribunal de Contas do Estado e o MP (Moreira et al., 2008; Pacheco et al., 2020; Shimizu et al., 2009; Zambon et al., 2013), com o propósito de possibilitar a avaliação e o acompanhamento dos resultados obtidos com as Organizações Sociais de Saúde, através de comparações entre aquilo que foi previamente estabelecido no contrato de gestão e os resultados alcançados (Pacheco et al., 2020).

Em acréscimo, segundo especificado na Constituição Federal de 1988, o MP é um componente fundamental no monitoramento de programas e recursos de saúde, proporcionando, assim, a atuação conjunta com os Conselhos de Saúde por meio de reuniões e debates (Aciole, 2003; Shimizu \& Dytz, 2009). Entretanto, estudos têm revelado uma relação frágil e superficial entre o MP e os Conselhos de Saúde em virtude da escassa participação dos promotores de justiça nas reuniões dos órgãos colegiados, prejudicando, assim, a orientação da política institucional do MP para que, através do diálogo interinstitucional, seja qualificado e fortalecido o controle social no SUS (Pereira et al., 2019).

Ainda sobre o funcionamento das instâncias deliberativas, para que os trabalhadores da saúde pudessem exercer a atribuição de conselheiros, as entidades que os representavam tinham que possuir sede local, prejudicando determinadas categorias profissionais, como a enfermagem que, apesar do elevado contingente de profissionais, não possuía representação na instância colegiada devido à falta de sede no município (Kezh et al., 2016).

De forma geral, quanto à escolha do presidente dos Conselhos de Saúde, verifica-se, em diferentes estudos, que os colegiados realizam a eleição presidencial em plenária (Moreira et al., 2008; Rocha et al., 2020; Silva et al., 2019; Vieira et al., 2011; Vilaça, Cavalcante et al., 2019; Zambon et al., 2011), nos quais são escolhidos presidentes do segmento de usuários (Vilaça et al., 2019), governo (Moreira et al., 2008) e secretários de saúde (Zambon et al., 2011). Entretanto, a contínua alternância da presidência dos Conselhos entre os "promotores da saúde" e os "usuários" tem tornado as práticas eleitorais pouco democráticas (Kezh et al., 2016), bem como a determinação do secretário de saúde como presidente nato em 77,7\% dos 27 Conselhos Estaduais de Saúde (CES) investigados no ano de 2005 (Escorel et al., 2008).

No tocante à escolha das instituições dos Conselhos, que deve ocorrer em reunião plenária através de indicação por seus pares, depreende-se que essa tem sido uma diretriz cumprida em determinadas instâncias colegiadas (Gerschman, 2004; Silva et al., 2019; Zambon et al., 2011), apesar de em outras prevalecerem critérios não eleitorais (Rocha et al., 2020), com renovação inclusive do quadro de conselheiros mediante a posse de um novo prefeito (Bispo Júnior et al., 2008).

Nesse sentido, verificam-se Conselhos de Saúde cujos membros são escolhidos através de indicação (Cotta et al.,2010), seja diretamente pelo prefeito (Cotta et al., 2009; Cotta et al., 2011; Zambon \& Ogata, 2013), secretário de saúde (Zambon et al., 2013) ou são indicados nas Conferências de Saúde (Gerschman, 2004). Tais práticas potencializam o conflito de interesses, ao passo que é enfraquecida a autonomia dos conselheiros de saúde (Zambon et al., 2013). Ademais, há órgãos colegiados que possuem membros designados pelas próprias entidades a que estão vinculados (Gerschman, 2004), através da diretoria ou da presidência das mesmas (Bispo Júnior et al., 2008).

No que diz respeito à estrutura administrativa e financeira dos Conselhos de Saúde, a pesquisa de Zambon e Ogata (2011) revela que metade das instâncias de deliberação investigadas possuía algum tipo de apoio administrativo e financeiro, 
cabendo ao órgão municipal de saúde esse suporte, enquanto a outra metade dos Conselhos, por não possuir tal apoio, apresentava fragilidades para o pleno desempenho de suas funções.

A existência de uma infraestrutura adequada (Shimizu et al., 2009), com a disponibilidade de linha telefônica, computador com acesso à internet, sala de reuniões, sede própria ou cedida pelo poder público (Gonçalves et al., 2008; Moreira et al., 2008; Rocha et al., 2020; Van Stralen et al., 2006; Vilaça et al., 2019), assim como a presença de apoio técnico e secretaria executiva, cuja função era desempenhada por um funcionário da própria secretaria de saúde, são fundamentais para o desenvolvimento das ações das instâncias participativas e na materialização do controle social no campo das políticas públicas de saúde (Escorel et al., 2008; Rocha et al., 2020; Silva et al., 2012; Vilaça, Cavalcante et al., 2019), embora existam Conselhos que não dispõem de infraestrutura adequada para reuniões, incluindo ventilação e iluminação (Escorel et al., 2008; Ventura et al., 2017), computador e internet, além de apoio técnico (Siqueira et al., 2015), linha telefônica exclusiva e sede própria (Escorel et al., 2008).

Não obstante seja estabelecido em Lei que cabe ao colegiado decidir sobre o planejamento e execução da sua dotação orçamentária (Brasil, 2012), observa-se que essa nem sempre é uma realidade dos canais institucionais de participação, visto que, ocasionalmente, os mesmos não a possuem ou simplesmente não têm autonomia para gerenciá-la, reduzindo, consequentemente, a viabilidade de intercâmbio com outros Conselhos e a participação dos conselheiros em eventos, de interesse para a coletividade, em outras localidades (Moreira et al., 2008; Rocha et al., 2020; Van Stralen et al., 2006; Ventura et al., 2017; Vieira et al., 2011).

A pesquisa de Escorel e Delgado (2008), por exemplo, revela que, em 2005, dos 27 CES pesquisados, 17 deles possuíam dotação orçamentária própria, sendo que apenas 7 apresentavam maior nível de autonomia financeira, pois além de possuírem a dotação orçamentária, também elaboravam o próprio orçamento e o gerenciavam. Nesse contexto, a pesquisa de Vilaça, Cavalcante e Moura (2019) evidencia que, no ano de 2017, o Conselho de Saúde do Distrito Federal possuía dotação orçamentária própria e, inclusive, a utilizava para realizar capacitação de seus membros.

Outro aspecto a ser considerado é a existência (ou não) de comissões internas nos Conselhos de Saúde que possuem como objetivo proporcionar que pautas específicas e complexas sejam monitoradas e abordadas de forma aprofundada por grupos familiarizados com determinadas temáticas (Rocha et al., 2020; Vieira et al., 2011). Na prática, percebe-se que tais comissões fazem parte dos espaços colegiados (Rocha et al., 2020; Silva et al., 2007), ainda que em determinados casos, as mesmas estejam presentes em menos da metade dos Conselhos Municipais de Saúde (Vieira et al., 2011). Dentre as experiências exitosas, a literatura reforça sobre episódios de reformulação e aglutinação das comissões, quando necessário, sendo considerada tanto a realidade local quanto o cenário para enfrentamento e efetivação das ações, possibilitando, assim, a otimização de gastos para a manutenção do funcionamento das diversas comissões (Silva et al., 2019).

Sob outra perspectiva, no que diz respeito à organização das Conferências de Saúde - ainda que seja uma diretriz prevista na legislação vigente - há uma quantidade significativa de Conselhos de Saúde que não definem a sua periodicidade, não examinam e propõem resoluções e deliberações após a conclusão das mesmas (Shimizu et al., 2009) e que simplesmente não a realizam (Zambon et al., 2013). Quanto ao funcionamento das Pré-Conferências de Saúde, verificou-se que tais espaços sociais - apesar da diversidade de público, com diferentes interesses (Lopes-Júnior et al., 2014) - são ideais para a intensificação da divulgação e escolha dos delegados para as Conferências de Saúde, representando uma oportunidade para que a população exerça o papel de protagonista frente ao controle social (Anunciação et al., 2008), mediante um amplo envolvimento da comunidade (Tofani et al., 2012).

Ainda sobre as Conferências de Saúde, Tofani e Carpintéro (2012) relatam a experiência do município de Várzea Paulista (SP) que encaminhou o relatório final de sua Conferência aos candidatos a prefeito, a fim de assegurar a sustentabilidade da política de saúde pactuada com a sociedade. A maioria das propostas foi organizada na forma de moções 
que foram encaminhadas também a diversos órgãos das esferas municipal e estadual. Essa experiência também contribuiu para o fortalecimento de espaços de debate entre os distintos sujeitos sociais, favorecendo a formação política dos participantes.

\section{Estrutura, funcionamento e organização das instâncias colegiadas informais}

Em relação às instâncias colegiadas informais, a exemplo das Comissões e Conselhos Locais de Saúde, verifica-se que as mesmas também eram orientadas através de regimento interno ou estatutos com normas específicas de funcionamento, os quais determinavam a duração dos mandatos dos conselheiros pelo prazo de dois anos (Lisboa et al., 2016; Oliveira et al., 2015), bem como estipulavam a distribuição percentual mínima de seus membros (Busana et al., 2015).

Complementarmente, eram realizadas reuniões ordinárias mensais e extraordinárias eventuais (Lisboa et al., 2016; Oliveira et al., 2009; Oliveira et al., 2015), apesar da convocação ser feita apenas com 24 horas de antecedência, havendo a discussão de diversos temas, tais como dengue, lixo, violência e eleições (Oliveira et al., 2015), cujas pautas, ocasionalmente, vinham prontas - sendo montadas antecipadamente pela secretaria do Conselho - ou eram elaboradas no início da reunião (Oliveira et al., 2009), existindo ainda espaços colegiados informais cujas pautas eram estabelecidas, via de regra, na reunião anterior, indicadas pelo próprio gerente da unidade de saúde que propunha temas prioritários (Oliveira et al., 2015).

Diehl e Langdon (2018) esclarecem que os Conselhos Distritais de Saúde Indígena devem manter um cronograma anual de reuniões destinadas à aprovação e avaliação dos programas de saúde, despesas e atividades propostas. No entanto, verificaram-se dificuldades organizacionais e financeiras para o seu atendimento.

De forma geral, no contexto da composição das instâncias informais, constata-se que as mesmas são compostas por segmentos de usuários e profissionais de saúde integrantes da própria unidade (Bispo Júnior et al., 2012; Busana et al., 2015; Lisboa et al., 2016) ou possuem composição tripartite, incluindo também representantes da gestão municipal (Arantes et al., 2007). Foi observado em Conselhos Gestores de Saúde o histórico de desrespeito à paridade de seus membros, que passou a ser respeitada apenas mais recentemente (Oliveira et al., 2009).

Quanto à escolha do presidente das instâncias informais, Oliveira e Almeida (2009) relatam em seu estudo que nos Conselhos Gestores Locais investigados, com o passar dos mandatos, percebeu-se que o segmento de prestadores de serviços passou a deter a coordenação dos Conselhos, cuja ocupação, anteriormente, ocorria prioritariamente pelo segmento de usuários.

Por sua vez, em relação à estrutura das Comissões Locais de Saúde, Oliveira e Dallari (2015) relatam que os colegiados investigados por elas não possuíam estrutura administrativa própria e funcionários que auxiliassem nas atividades. Dessa forma, atividades rotineiras, a exemplo da convocação para as reuniões - feita através de contato telefônico - eram realizadas por um conselheiro ou pelo próprio gerente da unidade de saúde.

\section{Estratégias e periodicidade de capacitação dos conselheiros de saúde nas instâncias colegiadas do SUS}

Os Conselhos de Saúde, compreendidos como espaços de pluralidade e diversidade na luta pela consolidação do direito à saúde e por democracia social (Aciole, 2003), devem incluir a participação de todos os seus membros no exercício deliberativo. Para isso, a literatura determina que apenas por meio da inclusão comunitária para o fortalecimento da cultura participativa e de uma contínua e permanente capacitação dos conselheiros de saúde, centrada na qualificação técnica e política - em especial entre os representantes dos usuários - será possível a aquisição de conhecimento, aumento da capacidade argumentativa, empoderamento dos sujeitos coletivos, mudança de práticas individuais e institucionais, compreensão do processo saúde-doença (subsidiando transformações no modelo de atenção à saúde), fomentando e consolidando a cultura de participação democrática e de resistência ao autoritarismo e clientelismos (ainda enraizados na cultura brasileira), assim como permitirá a superação de desafios das instâncias colegiadas, favorecendo uma prática qualificada e efetiva do controle social 
(Aciole, 2003; Aciole; 2007; Alencar, 2012; Batista et al., 2010; Bispo Júnior et al., 2012; Bispo Júnior et al., 2006; Bispo Júnior et al., 2008; Coelho, 2012; Cotta et al., 2009; Cotta et al., 2010; Cotta et al., 2011; Fernandes et al., 2017; Lisboa et al., 2016; Martinez et al., 2016; Martins et al., 2008; Moura et al., 2017; Pereira et al., 2019; Shimizu et al., 2009; Shimizu et al., 2015; Shimizu et al., 2013; Siqueira, Cotta, et al., 2015; Ventura et al., 2017; Zambon et al., 2013), pois "só se pode controlar aquilo que se conhece" (Cotta et al., 2009, p. 434) e "sem formação e informação não há poder de ação" (Duarte et al., 2012, p. 136).

Embora seja previsto na legislação vigente que cabe aos Conselhos de Saúde deliberar, elaborar, apoiar e promover a capacitação para o controle social, apenas 61,3\% dos Conselhos cadastrados no Sistema de Acompanhamento dos Conselhos de Saúde desenvolviam ações de educação permanente, com prevalências mais elevadas na região Norte, especialmente nos estados do Acre, Rondônia e Amapá, os quais apresentavam, respectivamente, 78,3\%, 77,5\% e 76,5\% de qualificação dos seus conselheiros de saúde (Da Silva et al., 2020).

Para Pereira et al. (2019), a indispensabilidade de uma política de capacitação em saúde deve ser direcionada também aos promotores de justiça do MP que, frequentemente, não possuem conhecimento técnico sobre gestão em saúde e/ ou saúde coletiva. Porém, é importante que os distintos sujeitos coletivos estejam dispostos e interessados a aprender, para que possam qualificar a execução das atribuições que lhes competem (Fakhouri et al., 2016; Oliveira et al., 2009).

Ademais, tais práticas formativas devem considerar as necessidades locais dos participantes, devendo ser pautadas no cotidiano dos mesmos, ou seja, com base na contextualização da realidade concreta, requerendo o emprego de práticas metodológicas que incluam a heterogeneidade da população em geral e dos diferentes integrantes dos Conselhos de Saúde (Alencar, 2012; Bispo Júnior et al., 2008; Da Silva et al., 2020; Shimizu et al., 2015; Ventura et al., 2017), priorizando o uso de metodologias capazes de potencializar a problematização das demandas dos conselheiros (Zambon et al., 2013) através de práticas expositivo-dialogadas, seminários temáticos, grupos de discussão, apresentação de filmes e oficinas que permitam a troca de informações, experiências e a consolidação de conhecimentos prévios dos participantes, além de materiais didáticos com linguagem de fácil compreensão e textos auxiliares para que esses atores coletivos alcancem condições de não somente compreender, mas também interferir no processo público decisório e de controle das políticas públicas de saúde (Alencar, 2012).

Apesar dessas informações norteadoras quanto à ideal prática formativa e a qual público ela se destina, estudos têm demonstrado que normalmente os projetos e cursos de capacitação, destinadas aos conselheiros de saúde, não levam em consideração as necessidades cotidianas, sobressaindo o emprego de práticas metodológicas e referenciais pedagógicos que, frequentemente, não contemplam a diversidade e heterogeneidade dos grupos (Ventura et al., 2017).

Outro aspecto a ser considerado é que - além dos conteúdos relacionados à saúde, legislações específicas e demais assuntos discutidos e deliberados nas instâncias colegiadas - as capacitações devem abranger a inclusão digital por intermédio dos ambientes virtuais de aprendizagem (Fernandes et al., 2017; Martinez et al., 2016; Oliveira et al., 2009; Silva et al., 2007), bem como considerar as parcerias com as instituições de nível superior para a capacitação tanto dos conselheiros como dos profissionais de saúde, sensibilizando-os para a atuação nos colegiados mediante uma abordagem centrada em ações interdisciplinares e debates críticos (Lopes-Júnior et al., 2014; Miranda et al., 2016; Oliveira et al., 2009).

Apesar dos conselheiros de saúde sentirem a necessidade de serem capacitados para atuar nos Conselhos (Cotta et al., 2009; Fernandes et al., 2017; Ventura et al., 2017) e embora tais instâncias compreendam a real necessidade das capacitações para um eficaz controle social e, por vezes, possuam o desejo de oferecê-las a seus membros (Arantes et al., 2007), sendo inclusive reivindicadas nas Conferências de Saúde (Miranda et al., 2016) e nas Pré-Conferências de Saúde (Lopes-Júnior, Pereira, \& Mishima, 2014), a maioria dos conselheiros nunca recebeu as referidas capacitações (Cotta et al., 2009; Duarte et al., 2012; Fernandes et al., 2017; Gonçalves et al., 2008; Ventura et al., 2017) ou apenas participaram de treinamentos 
esporádicos e pontuais, insuficientes para capacitá-los para atuarem nos Conselhos (Bispo Júnior et al., 2012), havendo inclusive experiências que demonstram a oferta de cursos de capacitação destinados aos membros de determinados espaços colegiados informais (Oliveira et al., 2013).

As explicações para a reduzida capacitação dos conselheiros de saúde estão relacionadas à expressiva rotatividade dos mesmos e/ ou devido à insuficiência de cursos direcionados à área do controle social (Duarte et al., 2012), sendo verificado na literatura que o escasso quantitativo de conselheiros que recebem capacitação - antes de assumirem a função - caracteriza uma fragilidade do colegiado, podendo ser explicada tanto pela baixa oferta de capacitações destinadas à participação social, conforme supracitado, quanto a um possível "efeito colateral" do dispositivo legal de rotatividade mínima dos membros do Conselho (Ventura et al., 2017). Todavia, é necessário compreender que as práticas de capacitação, antes de serem aplicadas, precisam ser discutidas para desconstrução e reconstrução com o outro, em especial nos ambientes em que predominam práticas excludentes e agressivas (Fakhouri et al., 2016).

No âmbito dos Conselhos Locais de Saúde, observa-se que o emprego de práticas pedagógicas, incluindo dinâmicas, atividades lúdicas e brincadeiras, facilita o processo de aprendizagem entre os partícipes, além de influenciar no fortalecimento das práticas comunitárias, intensificando o envolvimento popular nos microespaços e favorecendo, consequentemente, a sustentabilidade dos espaços colegiados de controle social local (Ponte et al., 2012).

Diante do exposto, torna-se essencial repensar as estratégias e a periodicidade das capacitações dos conselheiros de saúde, nas diferentes instâncias de participação social do SUS, como prioritárias para a consolidação do exercício do controle social na política de saúde, incluindo também os Conselhos Distritais de Saúde Indígena (Diehl et al., 2018), além da necessidade de outros estudos com a finalidade de elucidar como têm acontecido as ações de capacitação e quais os desafios a serem superados para sua efetivação (Da Silva et al., 2020).

\section{Conclusão}

Após três décadas de promulgação da Constituição Federal e de criação do SUS - considerado "a primeira política pública no Brasil a abarcar constitucionalmente a participação popular como um de seus princípios" (Busana et al., 2015, p. 447) - verificam-se avanços, desafios e insucessos do controle social no campo das políticas públicas em saúde. Essa realidade pode ser explicada pelos inerentes arranjos estruturais, organizativos e de funcionamento das instâncias colegiadas, formais e informais, nas três esferas governamentais, que se apresentam em processo de adequação quanto às diretrizes para seu funcionamento, estrutura e organização, conforme preconizado pelo Conselho Nacional de Saúde.

Nesse sentido, pode-se refletir a respeito das estratégias adotadas pelos órgãos colegiados que propiciam a seus membros uma atuação mais ativa, dinâmica e participativa nas deliberações em saúde pública, abdicando do desânimo e da inércia participativa, atuando, portanto, como protagonistas no processo decisório. É fundamental compreender, por exemplo, como as instâncias deliberativas têm realizado as capacitações permanentes de seus membros e qual a periodicidade em que elas são realizadas.

Tais práticas educativas, consideradas ferramentas estratégicas na potencialização do controle social, são assimiladas como responsáveis pela redução de assimetrias de poder entre os integrantes dos Conselhos de Saúde, pelo estímulo à participação consciente dos cidadãos, bem como pelo empoderamento desses sujeitos coletivos, atuantes no processo de construção e materialização do SUS, pois, segundo Escorel e Delgado (2008, p. 76), "a busca pela consolidação do SUS também envolve a consolidação da atuação dos Conselhos de Saúde".

Dessa forma, ressalta-se a necessidade da contínua educação permanente entre os conselheiros de saúde, visto que a mesma pode minimizar as dificuldades no exercício cotidiano de suas funções, além de potencializar a gestão das políticas públicas de saúde, na medida em que pode ser considerado um instrumento estratégico que fortalece a formação de uma 
consciência crítica e cidadã, possibilitando ainda aos diversos atores sociais uma participação efetiva, qualificada e que amplia o horizonte dos mesmos para além dos interesses próprios, ou seja, em defesa do bem comum.

Por fim, sugere-se que outros estudos sejam realizados para identificar a participação e efetividade do Controle Social na gestão do SUS, inclusive das ações intersetoriais, durante a pandemia da Covid-19, considerando ainda a (re)organização dos seus aspectos estruturais, organizativos e estratégicos.

\section{Referências}

Aciole, G. G. (2003). Algumas questões para o debate do controle social no Sistema Único de Saúde. Saúde debate, $27(63), 63-73$.

Aciole, G. G. (2007). Das dimensões pedagógicas para a construção da cidadania no exercício do controle social. Interface: Comunicação Saúde Educação, 11(23), 409-426.

Alencar, H. H. (2012). Educação permanente no âmbito do controle social no SUS: a experiência de Porto Alegre - RS. Saúde e Sociedade, 21(1), 223-233.

Anunciação, A. S. et al. (2008). Pré-Conferências de Saúde: uma proposta de democratização da saúde no município de Camaçari. Revista Baiana de Saúde Pública, 32(1), 131-137.

Arantes, C. I. S. et al. (2007). O controle social no Sistema Único de Saúde: concepções e ações de enfermeiras da atenção básica. Texto \& Contexto Enfermagem, 16(3), 470-478.

Assis, M. M. A. (2003). O processo de construção do controle social no Sistema Único de saúde e a gestão nos espaços institucionais. Saúde em Debate, 27(65), 324-335.

Batagello, R., Benevides, L., \& Portillo, J. A. C. (2011). Conselhos de Saúde: controle social e moralidade. Saúde e Sociedade, 20(3), 625-634.

Batista, A. A. et al. (2010). A contribuição da pesquisa avaliação para o processo de implementação do controle social no SUS. Saúde e Sociedade, 19(4), 784793.

Bispo Júnior, J. P., \& Martins, P. C. (2012). Envolvimento comunitário na Estratégia de Saúde da Família: dilemas entre institucionalização e efetiva participação. Physis: Revista de Saúde Coletiva, 22(4), 1313-1332.

Bispo Júnior, J. P., Messias, K. L. M., \& Sampaio, J. J. C. (2006). Exercício do controle social em municípios de pequeno porte: o caso de Lafayete Coutinho (BA). Revista Baiana de Saúde Pública, 30(2), 248-260.

Bispo Júnior, J. P., \& Sampaio, J. J. C. (2008). Participação social em saúde em áreas rurais do Nordeste do Brasil. Revista Panamericana de Salud Publica, 23(6), 403-409.

Botelho, L. L. R., Cunha, C. C., \& Macedo, M. (2011). O método da revisão integrativa nos estudos organizacionais. Revista Gestão e Sociedade. 5(11), 121136.

Brasil. Constituição (1988). Constituição da República Federativa do Brasil: promulgada em 5 de outubro de 1988 . Brasília: Senado Federal. http://www.planalto.gov.br/ccivil_03/constituicao/constituicao.htm

Lei $n^{o}$ 8.080, de 19 de setembro de 1990. Dispõe sobre as condições para a promoção, proteção e recuperação da saúde, a organização e o funcionamento dos serviços correspondentes e dá outras providências. Diário Oficial [da] República Federativa do Brasil, Brasília, DF, 20 set. 1990a. http://www.planalto.gov.br/ccivil_03/leis/18080.htm

Lei $n^{\circ}$ 8.142, de 28 de dezembro de 1990. Dispõe sobre a participação da comunidade na gestão do Sistema Único de Saúde (SUS) e sobre as transferências intergovernamentais de recursos financeiros na área da saúde e dá outras providências. Diário Oficial [da] República Federativa do Brasil, Brasília, DF, 31 dez. 1990b. http://www.planalto.gov.br/ccivil_03/leis/18142.htm

. Ministério da Saúde. Conselho Nacional de Saúde. Resolução n. 453, de 10 de maio de 2012. Aprova as seguintes diretrizes para instituição, $\begin{array}{llllll}\text { reformulação, reestruturação } & \mathrm{e} & \text { funcionamento } & \text { dos } & \text { Conselhos } & \text { de }\end{array}$ https://bvsms.saude.gov.br/bvs/saudelegis/cns/2012/res0453_10_05_2012.html

Busana, J. A., Heidemann, I. T. S. B., \& Wendhausen, A. L. P. (2015). Participação popular em um Conselho Local de Saúde: limites e potencialidades. Texto \& Contexto Enfermagem, 24(2), 442-449.

Coelho, J. S. (2012). Construindo a participação social no SUS: um constante repensar em busca de equidade e transformação. Saúde e Sociedade, 21 (1), 138151.

Cotta, R. M. M., Cazal, M. M., \& Martins, P. C. (2010). Conselho Municipal de Saúde: (re)pensando a lacuna entre o formato institucional e o espaço de participação social. Ciência \& Saúde Coletiva, 15(5), 2437-2445.

Cotta, R. M. M., Cazal, M. M., \& Rodrigues, J. F. C. (2009). Participação, controle social e exercício da cidadania: a (des)informação como obstáculo à atuação dos conselheiros de saúde. Physis: Revista de Saúde Coletiva, 19(2), 419-438.

Cotta, R. M. M. et al. (2010). Controle social no Sistema Único de Saúde: subsídios para construção de competências dos conselheiros de saúde. Physis: Revista de Saúde Coletiva, 20(3), 853-872. 
Cotta, R. M. M. et al. (2011). O controle social em cena: refletindo sobre a participação popular no contexto dos Conselhos de Saúde. Physis: Revista de Saúde Coletiva, 21(3), 1121-1138.

Da Silva, R. C. C., Novais, M. A. P., \& Zucchi, P. (2020). Permanent education as an inalienable responsibility of health councils: the current scenario in the Unified Health System. Clinics, 75, e.1443.

Diehl, E. E., \& Langdon, E. J. (2018). Indigenous participation in primary care services in Brazil: Autonomy or bureaucratization? Regions \& Cohesion, 8(1), 54-76.

Duarte, E. B., \& Machado, M. F. A. S. (2012). O exercício do controle social no âmbito do Conselho Municipal de Saúde de Canindé, CE. Saúde e Sociedade, 21(1), 126-137.

Durán, P. R. F., \& Gerschman, S. (2014). Desafios da participação social nos Conselhos de Saúde. Saúde e Sociedade, 23(3), 884-896.

Escorel, S., \& Delgado, M. M. (2008). Perfil dos Conselhos Estaduais de Saúde: singularidades e similaridades no controle social. Divulgação em Saúde para Debate, $43,62-78$.

Fakhouri, A. P., Francischetti, I., \& Vieira, C. M. (2016). Proposta de educação permanente para conselheiros municipais de saúde: relato de experiência. Revista Baiana de Saúde Pública, 40(4), 1029-1040.

Fernandes, V. C., Spagnuolo, R. S., \& Nascimento, E. N. (2017). Percepção de conselheiros de saúde sobre acesso às informações e inclusão digital. Saúde e Sociedade, 26(1), 218-228.

Gerschman, S. (2004). Conselhos Municipais de Saúde: atuação e representação das comunidades populares. Cadernos de Saúde Pública, 20(6), 1670-1681.

Gonçalves, A. O., Silva, J. F. S., \& Pedrosa, J. I. S. (2008). Contribuições da gestão participativa no espaço público chamado conselho: o contexto dos Conselhos de Saúde no Brasil. Divulgação em Saúde para Debate, 43, 96-105.

Gonçalves, R. S. et al. (2013). Conselhos de saúde e controle social: um estudo comparativo dos relatórios de prestação de contas das Secretarias Estaduais de Saúde. Saúde e Sociedade, 22(4), 1001-1013.

Kezh, N. H. P., Bógus, C. M., \& Martins, C. L. (2016). Entraves à participação social dos representantes dos trabalhadores no Conselho Municipal de Saúde. Saúde e Sociedade, 25(4), 950-963.

Labra, M. E. (2008). É possível aferir a qualidade da representação dos usuários nos Conselhos de Saúde? Divulgação em Saúde para Debate, $43,106-117$. Landerdhal, M. C. et al. (2010). Resoluções do Conselho de Saúde: instrumento de controle social ou documento burocrático? Ciência \& Saúde Coletiva, $15(5), 2431-2436$

Lisboa, E. A. et al. (2016). Conselhos locais de saúde: caminhos e (des)caminhos da participação social. Trabalho, educação e saúde, 14(3), 679-698. Lopes-Júnior, L. C., Pereira, M. J. B., \& Mishima, S. M. (2014). Participação popular e Pré-Conferência Municipal de Saúde. Revista da Rede de Enfermagem do Nordeste, 15(3), 543-553.

Magalhães, F. G. G. P., \& Xavier, W. S. (2018). Aspectos de autonomia e heteronomia no exercício do controle social em um Conselho Municipal de Saúde. Cadernos Gestão Pública e Cidadania, 23(75), 261-283.

Martinez, M. G., \& Kohler, J. C. (2016). Civil society participation in the health system: The case of Brazil's Health Councils. Globalization and Health, 12(64), 1-12.

Martins, P. C. et al. (2008). Conselhos de Saúde e a participação social no Brasil: matizes da utopia. Physis: Revista de Saúde Coletiva, 18(1), 105-121

Miranda, A. S. et al. (2016). Discursos práticos sobre ocorrências, processos decisórios e decorrências de Conferências Municipais de Saúde. Interface: Comunicação Saúde Educação, 20(58), 559-571.

Mittelbach, J. C. S., \& Perna, P. O. (2014). A percepção dos enfermeiros sobre o seu papel nos Conselhos de Saúde enquanto segmentos dos trabalhadores de saúde. Cogitare Enfermagem, 19(2), 284-291.

Moreira, M. R. et al. (2008). Participação nos Conselhos Municipais de Saúde de municípios brasileiros com mais de cem mil habitantes. Divulgação em Saúde para Debate, 43, 48-61.

Moura, L. M., \& Shimizu, H. E. (2017). Representações sociais de saúde-doença de conselheiros municipais de saúde. Physis: Revista de Saúde Coletiva, 27(1), 103-125.

Müller Neto, J. S., \& Artmann, E (2014). Discursos sobre o papel e a representatividade de conferências municipais de saúde. Cadernos de Saúde Pública, $30(1), 68-78$.

Moher, D. et al (2009). The PRISMA Group 2009. Preferred reporting items for systematic reviews and meta-analyses: the PRISMA Statement. PLOS Medicine, 6(6), e.1000097.

Oliveira, V. C. (2004). Comunicação, informação e participação popular nos Conselhos de Saúde. Saúde e Sociedade, 13(2), 56-69.

Oliveira, M. L., \& Almeida, E. S. (2009). Controle social e gestão participativa em saúde pública em unidades de saúde do município de Campo Grande, MS, 1994-2002. Saúde e Sociedade, 18(1), 141-153.

Oliveira, A. M. C., \& Dallari, S. G. (2015). Participação social no contexto da Atenção Primária em Saúde: um estudo de caso das Comissões Locais de Saúde do SUS de Belo Horizonte. Physis: Revista de Saúde Coletiva, 25(4), 1059-1078.

Oliveira, A. M. C., Ianni, A. M. Z., \& Dallari, S. G. (2013). Controle social no SUS: discurso, ação e reação. Ciência \& Saúde Coletiva, 18(8), $2329-2338$. 
Pacheco, H. F. et al. (2020). A accountability das Organizações Sociais no SUS: uma análise do papel institucional do Conselho Estadual de Saúde em Pernambuco. Physis: Revista de Saúde Coletiva, 30(1), e.300108.

Pereira, I. P. et al (2019). Ministério Público, Conselhos Municipais de Saúde e as práticas do diálogo interinstitucional. Saúde e Sociedade, 28(2), 111-123.

Pessoto, U. C., Nascimento, P. R., \& Heimann, L. S. (2001). A gestão semiplena e a participação popular na administração da saúde. Cadernos de Saúde Pública, 17(1), 89-97.

Ponte, H. M. S. et al. (2012). "O caminho se faz ao caminhar": processo de reativação de Conselhos Locais de Saúde em Sobral, a partir do protagonismo cidadão. Saúde e Sociedade, 21(1), 234-243.

Rocha, M. B., Moreira, D. C., \& Bispo Junior, J. P. (2020). Conselho de saúde e efetividade participativa: estudo sobre avaliação de desempenho. Cadernos de Saúde Pública, 36(1), e00241718.

Santos, C. L. et al. (2020). Os Conselhos de Saúde e a publicização dos instrumentos de gestão do SUS: uma análise dos portais das capitais brasileiras. Ciência \& Saúde Coletiva, 25 (11), 4389-4399.

Serapioni, M., \& Romaní, O (2006). Potencialidades e desafios da participação em instâncias colegiadas dos sistemas de saúde: os casos de Itália, Inglaterra e Brasil. Cadernos de Saúde Pública, 22(11), 2411-2421.

Severo, D. O., \& Da Ros, M. A. (2012). A Participação no controle social do SUS: concepção do Movimento dos Trabalhadores Rurais Sem Terra. Saúde e Sociedade, 21(1), 177-184.

Shimizu, H. E., \& Dytz, J. L. (2009). Local health governance in central Brazil. Journal of Ambulatory Care Management, 32, 131-139.

Shimizu, H. E., \& Moura, L. M. (2015). As representações sociais do controle social em saúde: os avanços e entraves da participação social institucionalizada. Saúde e Sociedade, 24(4), 1180-1192.

Shimizu, H. E. et al. (2013). Representações sociais dos conselheiros municipais acerca do controle social em saúde no SUS. Ciência \& Saúde Coletiva, 18(8), $2275-2284$

Silva, A. X., Cruz, E. A., \& Melo, V (2007). A importância estratégica da informação em saúde para o exercício do controle social. Ciência \& Saúde Coletiva, 12(3), 683-688.

Silva, L. M. S. et al. (2012). Análise da organização e funcionamento dos Conselhos de Saúde e a gestão participativa em Fortaleza, CE. Saúde e Sociedade, 21(1), 117-125.

Silva, B. T., \& Lima, I. M. S. O (2019). Análise política da composição do Conselho Nacional de Saúde (2015/2018). Physis: Revista de Saúde Coletiva, 29(1), e290113.

Siqueira, R. L., Cotta, R. M. M., \& Soares, J. B. (2015). Conhecimentos estratégicos para a participação social no Sistema Único de Saúde. $O$ Mundo da Saúde, 39(1), 32-42.

The Joanna Briggs Institute (2014). Joanna Briggs Institute Reviewers’ Manual: 2014 edition. Adelaide: The Joanna Briggs Institute.

Tofani, L. F. N., \& Carpintéro, M. C. C. (2012). $3^{\text {a }}$ Conferência Municipal de Saúde de Várzea Paulista: a participação da sociedade no processo de priorização e compromisso político. Saúde e Sociedade, 21(1), 244-252.

Van Stralen, C. J. et al. (2006). Conselhos de Saúde: efetividade do controle social em municípios de Goiás e Mato Grosso do Sul. Ciência \& Saúde Coletiva, 11(3), 621-632.

Ventura, C. A. A. et al. (2017). Cultura participativa: um processo de construção de cidadania no Brasil. Interface: Comunicação Saúde Educação, 21(63), 907-920.

Vieira, M., \& Calvo, M. C. M. (2011). Avaliação das condições de atuação de Conselhos Municipais de Saúde no Estado de Santa Catarina, Brasil. Cadernos de Saúde Pública, 27(12), 2315-2326.

Vilaça, D. S. S., Cavalcante, D. S., \& Moura, L. M. (2019). Atuação do Conselho de Saúde do Distrito Federal na reforma da Atenção Primária à Saúde, de 2016 a 2018: estudo de caso. Ciência \& Saúde Coletiva, 24(6), 2065-2074.

Zambon, V. D., \& Ogata, M. N. (2011). Configurações dos Conselhos Municipais de Saúde de uma região no Estado de São Paulo. Revista da Escola de Enfermagem da USP, 45(4), 890-897.

Zambon, V. D., \& Ogata, M. N. (2013). Controle Social do SUS: o que pensam os conselheiros municipais de saúde. Revista Brasileira de Enfermagem, Brasília, 66(6), 921-927. 